\title{
Special issue: Sign language translation and avatar technology
}

\author{
Rosalee Wolfe ${ }^{1}$
}

Published online: 8 June 2021

(c) The Author(s), under exclusive licence to Springer Nature B.V. 2021

Members of Deaf communities around the world face significant barriers to education, employment, and health care. They need efficient, affordable translation in order to gain access to services that in the hearing world are taken for granted. For this reason, automatic translation between signed and spoken languages promises substantial benefits.

Many people are surprised to learn that the primary communication barrier for the deaf ${ }^{1}$ is not sound but language. A deaf community uses a signed language as the first or preferred language. Signed languages are natural languages, as fully formed as any spoken language and independent of the language of the country's hearing community. The differences between a signed and a spoken language are greater than the differences between any two spoken languages due to the contrast in the communication channels. Moreover, like spoken languages, signed languages vary from one geographic region to another. Consequently, creating a translator between a signed language and a spoken language is at least as challenging as creating a translator between spoken languages.

\section{The challenge}

Automatic spoken/signed language translation presents far more hurdles than those encountered in conventional spoken/spoken translation. These include

- Differences in modality

- Absence of a standard written form

- Scarcity of resources

1 The term "deaf" as used here refers to groups of people who share a common signed language and culture. This is in contrast to another meaning of the term that refers to the audiological condition of not hearing.

Rosalee Wolfe

rwolfe@depaul.edu

1 School of Computing, DePaul University, Chicago, IL, USA 


\subsection{Differences in modality}

Speech utilizes the vocal apparatus for production, and hearing for reception. Spoken languages use visible communicative behaviors as well, but speakers can produce and comprehend audio-only sources and written language. In contrast, signed languages use only visible actions for production, and vision for reception. Furthermore, where speech utilizes phonation for producing utterances - a single vibrating column of air-signed languages use the configuration and movement of multiple body parts concurrently, including hands, head, face, eyes, and torso. A change in any of these, or a change in timing, can cause a change in meaning.

This is analogous to the difference between a solo instrument and an orchestra. The clarinet, for example, is like the speaking voice, capable of a wide range of expression through variations in pitch, tempo, dynamics, and phrasing. In contrast, signed language is performed by an entire ensemble of articulators, each with pitch, tempo, dynamics, and phrasing. As with an orchestral performance, errors by a single member of this ensemble can interfere with the message to the audience.

The complexity of sign language production poses significant problems for computer recognition strategies, including occlusion, signer-dependent variations, and the task of extracting features from a background. Hands can pass in front of the face, obscuring it from the viewer. One hand can disappear behind the other and reappear milliseconds later. Signer-dependent variations arise when the same utterance is produced by people of differing heights and physiques. The challenge of extracting features is exacerbated when the background behind a signer is complex or when the lighting changes.

\subsection{Absence of a standard written form}

Automatic systems for speech translation rely on written forms of the source and target languages. It is not possible to apply this type of technology directly to signed languages because signed languages do not have a widely accepted written form. Although some signs correspond to single words or phrases in a spoken language, much of signed language cannot be captured in a dictionary or concordance.

In spoken languages, phonetic elements are perceived as sequential; in signed languages, these elements are often simultaneous. Techniques for representing co-occurring elements have progressed substantially in the past 20 years, but there are still challenges in expressing prosodics, spatio-temporal modifiers, and low-level kinematics.

In the absence of a writing system, the target language must be displayed through avatar technology. Spliced video clips are not a viable alternative because a video clip is a slice in time: The act of creating clips will sever co-occurring processes that overlap in time. Readable display of signed language through avatar technology requires a representation system that can fully capture processes that are co-occurring at each linguistic level. 


\subsection{Scarcity of resources}

Signed languages are under-resourced for software tools and data. Signed language corpora are small in comparison with those for spoken language, and creating or expanding corpora is expensive. This makes corpus-based translation difficult. Aggravating the situation even further is that few computer-assisted translation tools are available to human interpreters and translators. At this early stage of avatar technology, moreover, signing still often looks robotic. Reading such awkward motion is at best frustrating, often overwhelming.

\section{The promise: moving forward}

A lively, engaged community of researchers is grappling with these challenges. They meet periodically at workshops in Sign Language Translation and Avatar Technology (SLTAT) to discuss new approaches and share insights. The papers in this issue derive from work first presented at the fifth workshop, held in Hamburg, Germany in October, 2019.

The first set of papers in this issue discusses two strategies for improving signedlanguage resources. Kaczmarek and Filhol report on efforts to create better tools for human translators, which can in turn augment corpora. In Computer-assisted Sign Language translation: a study of translators' practice to specify CAT software, they describe an initial study to identify translator needs for producing fast, accurate translation. In the second paper, Sign boundary and hand articulation feature recognition in Sign Language videos, Koulierakis et al. present a recommendation system for automatic annotation of sign language video, an important step toward building larger signed corpora.

The second set of papers examines approaches to advancing sign language representation and its application. In Animating adverbs of manner, Moncrief describes the representation of a class of non-lexical modifiers that manifest through changes in manner of motion. Next, Natural synthesis of productive forms from structured descriptions of sign language, by McDonald and Filhol, proposes an expanded representation to facilitate signed discourse via avatar. Utilizing higher-level language structures to support the production of lower-level structures results in more natural, smoothly flowing motion.

The final set of papers addresses the challenge of improving sign language production through better avatar animation. Baowidan describes a robust technique for collision avoidance in Improving realism in automated fingerspelling in American Sign Language with the potential for generating fingerspelling in all sign languages that use a one-handed manual alphabet. Naert et al. advance the reuse of motion capture in Motion synthesis and editing for the generation of new sign language content: Building new signs with phonological recombination. Their approach opens up new avenues for both creating richer corpora and using motion capture to produce novel utterances while preserving co-occurring processes. In the final paper, Towards enhanced visual clarity of sign language avatars through recreation of fine facial detail, Johnson discusses modifications to classic techniques of computer 
graphics to enable accurate portrayal of facial signals, a vital feature of signed language avatars that is often neglected.

These papers represent new inroads into the three challenges-a different modality, the absence of a standard written form, and the scarcity of language resources. The editors hope that these will prove helpful for future researchers and inspire them to work toward the goal of better Deaf/hearing communication through automatic translation.

Chicago, IL

May 26, 2021

Publisher's Note Springer Nature remains neutral with regard to jurisdictional claims in published maps and institutional affiliations. 